FARNER und KIINGER, daß dies bei Ratten und Katzen nicht immer der Fall sein muß. Latente Tetanie kann nach ihnen beim Tier (Schreck, Ubberanstrengung) zum blitzartig eintretenden ,Tetanus" ausarten; dieser geht entweder zurück oder endet mit dem Tode (vgl. ERDHerm) ${ }^{1}$ ).

Nach Strumaoperation kann man beim Menschen öfter leichte Symptome von Tetanie nachweisen (CHvoster), wenn man besonders darnach fahndet (LOBENHOFFER, MELchior). v. EISELSBERG und MELCHIOR wiesen ausdrücklich darauf hin, daß man mit der Bezeichnung ,leicht" sehr vorsichtig sein muß. Die Möglichkeit, daß bei den Kranken später eine akute, ja tödliche Form auftritt, ist gegeben.

Ich sah beim Menschen zwei tödliche Fälle, bei diesen war nur eine Seite operativ angegangen worden! Pfötchenstellung, Chvostek war einige Male zu bemerken, namentlich im Frühjahr (vgl. Mor, Der Frühlingsgipfel der Tetanie. M. m. W. r9I9). Die Erscheinungen gingen aber bald zurück und blieben weg, wenigstens bisher. Es besteht in Heidelberg, Wien und andernorts eine gewisse Tetaniebereitschaft; die Kriegsfolgen mögen sie erhöht haben. MELCHIOR sah Tetanie nach einer Operation wegen Kryptorchismus, wir nach Operation eines Halluse valgus!

Als Therapie kommt hauptsächlich (v. EiselsBerg) die medikamentöse in Betracht:

I. Parathyreoidintabletten,

2. Calcium lacticum bis $30 \mathrm{~g}$ am Tag,

3. Afenil intravenös (v. EIselssberg),

4. Chloralhydrat-Klysma,

5. Mehlfreie Kost (Fuchs, v. Eiselsberg).

Mc. Callum sagt, daß diese Behandlung nur symptomatisch ist; sie ,maskiert" nur den Ausfall der Epithelkörperchen. Man kann sich aber u. E. wóhl vorstellen, daß sie bei nur geschädigten Organen dem Körper über die Krise bis zur Erholung hinweghilft.

Versagen die eben erwähnten Mittel, so ist der Versuch der E. K.-Transplantation gerechtfertigt. Nach unseren jetzigen Anschauungen ist es nicht erlaubt, bei der Kropfoperation eines zu entnehmen; man weiß nie bestimmt, wieviele der unfreiwillige Spender noch hat. Man wird frisch Verunglückte, eben Gestorbene wählen. Bei Kindern, die während der Geburt starben, sind die kleinen Organe schwer zu finden. Beim Erwachsenen empfiehlt es sich dringend, eine mikroskopische Untersuchung des Pfröpflings vorzunehmen, da sonst ein Irrtum zu leicht möglich ist. v. EISELSBERG fand in der Literatur mehr als 20 Transplantationen vom lebenden Menschen angeführt, von welchen 20 einheilten. Die Wirkung ist schwer zu übersehen, meint er, da bei vielen voreilig transplantiert wurde. Borchers ist weniger skeptisch. Ich transplantierte vor mehr als 2 Jahren wegen Tetanieepilepsie (keine Operation vorausgegangen). Seither ist der Kranke frei von seinen Anfällen. Nachdem, was ich bisher von Homoiotransplantation sah, kann ich von einem Dauererfolg nicht sprechen. Auch v. EISELSBERG schreibt, da $\beta$ von einem einwandfreien bleibenden Einheilen $z$. Zt. noch nicht die Rede sein könnte.

Die Kropfoperation ist nach all dem Angeführten nicht absolut gefahrlos. Die Träger der Struma sind sich auch zum Teil darüber im klaren. Außer der Frage: Ist Gefahr vorhanden, werde ich nicht blödsinnig, wird uns auch oft die vorgelegt, ob der Kropf nicht wieder kommt. Die Antworten lauten der Literatur nach äußerst verschieden (vgl. unten). Sie können unmöglich alle Platz finden. Die Anschauung von JUL. WoLfF, $\mathrm{da} B$ nach der Operation der einen Seite die andere zum Schwinden komme, wird kaum noch vertreten. Der Glaube von STRÄUlI, daß die Verlagerung des Kropfrestes ein Rezidiv verhüte, ist durch die Mitteilungen von v. HoFMeISTER bedenklich erschüttert worden. TH. KocHER glaubte die Wiederkehr durch seine Enukleationsresektion vermeiden zu können, Kausch durch die Resektion nach Mikulicz, HoTz und ENDERLEN hoffen von der ausgiebigen Reduktion Gutes ${ }^{1}$ ).

Nun zu den Antworten: Sehr optimistisch ist HrLdeBRAND, der ,gewöhnlich die Hemistrumektomie übt". Unter seinen Berliner Fällen sah er Rezidive bzw. Strumaentwicklung auf der anderen Seite „,außerordentlich selten“. Roux ist Pessimist: Der Patient steigt mit dem Rezidiv vom Operationstisch herunter; weiter: er ist ein Kandidat des Rezidives, wenn man ihm 2 Lappen läßt, mehr oder weniger zugestutzt. Er ist nur geschützt, wenn er in einem gewissen Alter ist, in dem man Philosoph geworden ist und die Gelegenheit hat, vorher an einer anderen Kankheit zu sterben, ehe ihn sein Kropf wieder belästigt".

Roux wurde in diesem wenig günstigen Urteil durch die Arbeit seines Schülers GERMANIER bestärkt. Auch noch nach 25 Jahren konnte er ein Rezidiv feststellen (BRUNNER nach $3^{\circ}$ Jahren). Ich greife die niederste und höchste errechnete Zahl heraus: Halbseitenreseltion 25\%, Enucleoresektion $39,4 \%$; BrunNer rechnet in $3 \mathrm{I} \%$; PALLA in $26 \%$, Bergat $65 \%$, NÄGELI in $4,1 \%$ auf Wiederkehr der Schwellung. Auf weitere Angaben verzichte ich.

Diejenigen Autoren, welche auf kurze Zeit zurückgreifen, erhalten die niedersten Zahlen.

Herr Dr. Hrtzler prüft die Rezidive der Heidelberger Klinik z. Zt. nach (1897-I9I5). 795 Patienten konnten entwedernachuntersucht werden oder sie gaben Auskunft. Soviel sich bis jetzt übersehen läßt, handelt es sich um 234 (29\%) echte Rezidive und um I $_{20}$ ( I $5 \%$ ) falsche (Vergrößerung der unberührten Seite).

Mit der gebotenen Vorsicht möchte ich aus den bisherigen Feststellungen mit anderen annehmen, daß es sich beim Wiederwachsen des Kropfes nicht nur um die Art der Operation, sondern auch um die Art der Struma handelt.

Die Kropffrage ist noch nicht erledigt.

\title{
ORIGINALIEN.
}

\section{ÜBER DIE KLINISCHE PULSUNTERSUCHUNG BEI PATIENTEN MIT UNREGELMÄSSIGEM PULS, NAMENTLICH BEI ARHYTHMIA PERPETUA.}

\section{Von}

Privatdozent Dr. med. Christen LundsGaARd, Oberarzt. Aus der medizinischen Klinik d. Univ. Kopenhagen (Prof. Dr. med. KNUDFABER).

\section{Einleitung.}

Von vielen Ärzten wird die Differentialdiagnose zwischen den verschiedenen Arhythmieformen als eine so schwierige Sache betrachtet, daß sie ganz den Spezialisten oder Krankenhäusern überlassen werden muß, wo es möglich ist, elektrokardiographische oder sphygmographische Untersuchungsmethoden zu benutzen. Diese Auffassung trifft in der Tat nicht zu. Nachdem wir durch die neueren exakten Methoden

1) Die Tetanie ist nach FARNER und KLINGER eine Vergiftung mit Basen der Guanidingruppe; E. FRANK und seine Mitarbeiter denken daran, daß die tetanogenen Stoffe dem Dimethylgianidin nahe stehen. zur Untersuchung des Rhythmus des Herzens über die verschiedene Pathogenese und die Außerungsformen der verschiedenen Arhythmien unterrichtet worden sind, zeigt es sich, daß mehrere - oft altbekannte - allgemeine klinische Symptome sich aus unserer tieferen Einsicht in den Mechanismus des Herzens bei diesen krankhaften Verhältnissen erklären lassen und zu verstehen sind. Ferner zeigt eine nähere Beobachtung, daß viele von diesen Symptomen so charakteristisch für bestimmte Leiden sind, daß sich auf Grund der klinischen Svmptome allein oft eine zuverlässige Diagnose stellen läßt.

Die gegenwärtige Abhandlung enthält eine Darstellung des sogenannten „Pulsdefizites" mit besonderer Berücksichtigung seiner Bedeutung für die Diagnose und Behandlung der Arhythmia perpetua.

Wenn die Herzaktion unregelmäßig vonstatten geht, wie bei Patienten mit Vorkammerflimmern und daraus folgender Arhythmia perpetua, werden die Ventrikel sich in verschie-

1) Die Zeit muB lehren, ob diese Hoffnung nicht trügt. Die Herren ROUX und HEDINGER sprachen ihre Bedenken mir gegenüber aus. 
denem Grade füllen. Der Puls wird daher nicht nur irregulär, sondern auch inäqual, indem größere und kleinere Schlagvolumina sich ablösen. Oft sind verschiedene von ihnen so klein, daß die durch sie hervorgerufene Pulswelle sich verliert, bevor sie das Handgelenk erreicht, so daß sie sich nicht palpieren läßt. Mitunter werden nicht einmal die Aorta(und Pulmonal)-Klappen geöffnet, so daß die Ventrikel in einem solchen Falle nur eine isometrische Kontraktion um den Inhalt ausführen. In beiden Fällen wird die Frequenz des Herzens höher liegen als die des Radialispulses. Dieser Unterschied zwischen der Frequenz des Herzens und des Pulses, der eine Folge der inäqualen Schlagvolumina und eine indirekte Folge von unregelmäßiger Herztätigkeit darstellt, ist ein altbekanntes Symptom bei gewissen Herzleiden, das jedoch erst seit neuerer Zeit in dem Grade beachtet worden ist, daß man es der Mühe wert gefunden hat, den Unterschied bei dem einzelnen Patienten besonders zu untersuchen und aufzuzeichnen.

Den Anfang machten im Jahre I9I2 RoBInson und DRAPER, die (im Rockefeller Hospital) eine Reihe von Vergleichungen zwischen der Frequenz des Herzens und der Häufigkeit des Pulses bei Patienten mit unregelmäBigem Puls anstellten. Sie kennzeichneten den Unterschied durch den Terminus ,Pulsdefizit". Thre Untersuchungen sind jedoch nie veröffentlicht worden. Im Jahre I9I 4 erschien eine kurze Publikation über die Frage von JAmes und HART ${ }^{1}$ ), welche Robinson und Drapers Idee und den Namen „Pulsdefizit“ im weiteren Kreise einführte. Während ein einfacher zahlenmäßiger Nachweis des Pulsdefizites bei Patienten mit Atriumflimmern mehrfach in Amerika Anwendung gefunden hat, hat er in Europa nicht durchzudringen vermocht. Dies beruht wahrscheinlich darauf, daß die Frage in der Literatur so wenig erörtert worden ist, und ist sehr zu bedauern, da die Methode in der Tat einen bedeutenden klinischen Wert besitzt und zu einem wertvollen Hilfsmittel bei der Diagnose und der Behandlung der Arhythmia perpetua herangebildet werden kann.

Die Methodik ist äußerst einfach und erfordert, von einem Stethoskop und einer Uhr abgesehen, kein Instrumentarium. Am besten, was jedoch nicht durchaus notwendig ist, wird die Untersuchung von 2 Personen ausgeführt, von denen die eine das Herz auskultiert, die andere den Puls zählt. Die Uhr wird so angebracht, $\mathrm{da} B$ beide Untersucher sie sehen können. $\mathrm{Zu}$ einem im voraus verabredeten Zeitpunkt, z. B. zu Anfang einer Minute, beginnen beide Untersucher zu zählen und fahren $1 / 2$ Minute damit fort. Weiterer Sicherheit halber kann der eine den Anfang durch das Austufen von ,eins" und den AbschluB durch das Aussprechen der von ihm erreichten Endzahl kennzeichnen. Danach richtet sich der andere Untersucher. Die Untersuchung läßt sich jedoch auch durch eine Person bewerkstelligen, wobei die Herzfrequenz und der Puls in zwei aufeinander folgenden Zeiträumen zu zählen sind, und zwar, um Zufälligkeiten zu vermeiden, eine ganze Minute lang. Dies gilt jedoch nur bei Patienten in Ruhe. Nach Arbeit, z. B. Treppensteigen, muß die Herz- und die Pulsuntersuchung gleichzeitig stattfinden, da die durch die Arbeit hervorgerufene Tachycardie nach dem Aufhören der Anstrengung mehr oder minder schnell abnimmt, aus welchem Grunde man hier auch eine bestimmte Zeit (1/2 Min.) lang zählen $\mathrm{mu} \beta$.

Tab. I enthält Zählungen von I 8 verschiedenen an Arhythmia perpetua leidenden Patienten. Es handelt sich in allen Fällen um die erste Zählung, die bei der Mehrzahl während der ersten Tage ihres Krankenhausaufenthaltes und vor der medikamentalen Behandlung ausgeführt wurde. Es wurde gleichzeitig von 2 Untersuchern $1 / 2$ Min. lang gezählt. Das Pulsdefizit schwankt zwischen 2 (Nr. 8) und 82 (Nr. II). Die Tabelle zeigt, daß ein Zählen des Pulses allein einen falschen Eindruck von der Arbeitsweise des Herzens erzeugt. Der Puls schwankt zwischen 96 und 62 , also im wesentlichen innerhalb normaler Werte. Erst ein Zählen der Herzfrequenz unterrichtet uns von der sogar sehr bedeutenden Tachycardie der meisten dieser Patienten, indem die Herzfrequenz zwischen 72 (Nr. 8) und I72 (Nr. II) schwankt²). Dies zeigt also, daß i) W. B. JAMES and T. S. HART, Auricular Fibrillation; Clinical Observations on Pulse Deficit, Digitalis and Blood-pressure. Americ. Journ. of the Med. Sciences 147, 63. 1914 .

2) So findet sich das kleinste Pulsdefizit bei demjenigen Patienten, der die langsamste Hetzaktion aufweist, wāhrend das größte Pulsdefizit in Verbindung mit der schnellsten Herzaktion vorkommt. es bei Patienten mit Arhythmia perpetua nicht angeht, sich mit einer Pulszählung zu begnügen (siehe Tab. I).

Tabelle I.

Pulsdefizit in Ruhe bei I 8 Patienten mit Arhythmia perpetua. Die Zählungen fanden in den meisten Fällen unmittelbar nach der Aufnahme statt.

\begin{tabular}{|c|c|c|c|c|c|c|c|c|c|}
\hline 究 & Name & 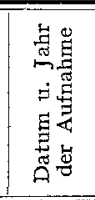 & 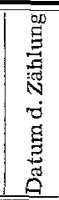 & 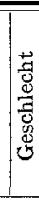 & 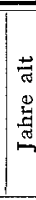 & 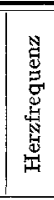 & 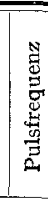 & 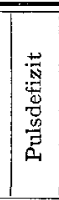 & $\begin{array}{c}\text { Diagnose ausschließlich der } \\
\text { Arhythmie }\end{array}$ \\
\hline & J. S. & $5 / 818$ & $19 / 8$ & 오 & 26 & 120 & 84 & & Insuff. et stenosis mitralis. \\
\hline & $\mathrm{J}, \mathrm{R}$. & $4 / 10^{18}$ & $5 / 10$ & 0 & 26 & 132 & 66 & 66 & Insuff. mitralis. \\
\hline & J. G. & $28 / 12^{18}$ & $30 \%$ & 오 & 44 & $7^{8}$ & 64 & I4 & Insuff. et stenosis mitralis. \\
\hline & J.P,H. & & $9 / 1$ & $0^{\top}$ & 46 & 90 & 78 & I 2 & Stenosis mitralis. \\
\hline 5 & A. $\mathrm{H}$. & $29 \% 1$ i & $30 \%$ & 우 & 36 & 142 & 78 & 64 & $\begin{array}{l}\text { Stenosis mitralis. Endo- } \\
\text { carditis recurrens }\end{array}$ \\
\hline 6 & S. L, & $15 / 3$ I9 & $16 / 3$ & & 22 & $\mathrm{I} 28$ & 96 & 32 & Insuff. et stenosis mitralis. \\
\hline & O. B. & $11 / 419$ & $22 / 4$ & 오 & 33 & 130 & 88 & 42 & Insuff. mitralis. \\
\hline & A.M.P, & & $16 / 7$ & 우 & 25 & 72 & 70 & 2 & Insuff. mitralis. Endo- \\
\hline & C.V.O. & $20 / 719$ & $21 / 7$ & & 51 & IIO & 62 & 48 & Stenosis mitralis. \\
\hline & J. H. & & $6 / 8$ & & 43 & 122 & 66 & 56 & Stenosis mitralis. \\
\hline II & S. C. & $12 / 819$ & $13 / 8$ & 오 & 37 & 172 & 92 & 82 & Insuff. et stenosis mitralis. \\
\hline I2 & $\mathrm{O}, \mathrm{C}$ & $21 / 9$ I9 & $25 \%$ & & 29 & 94 & 84 & 10 & Insuff. et stenosis mitralis. \\
\hline 13 & E. N. & $8 / 120$ & $10 \%$ & 오 & 37 & I 34 & 80 & 54 & Stenosis mitralis. \\
\hline 14 & D. S. & $20 \% 420$ & $4 / 5$ & 오 & 35 & 92 & 66 & 26 & Stenosis mitralis. \\
\hline 15 & W. S. & $15 / 121$ & $17 / 1$ & 0 & 59 & ${ }^{2} 5^{2}$ & 72 & 80 & Hypertensio arterialis. \\
\hline I6 & C. H. & $12 / 821$ & $15 / 8$ & & 45 & 130 & 80 & 50 & Oedema pulm. \\
\hline I 7 & V.E,N & $13 \%$ & $13 \%$ & 오 & 25 & 126 & 96 & 30 & Insuff. mitralis. \\
\hline I 8 & & & $29 / 9$ & Q & & 120 & 75 & 45 & Stenosis mitralis. \\
\hline
\end{tabular}

Tab. I liefert im übrigen ein Beispiel von dem häufigen Zusammentreffen von Arhythmia perpetua und den Mitralfehlern. Bei 16 von I8 Patienten lagen entweder Mitralstenose oder Mitralinsuffiziens oder auch beide Fehler vor. In einem Falle zugleich Aortainsuffiziens. Bei 2 Patienten, Nr. I5 und I6, von denen der eine Hypertonie aufwies, fand sich kein Klappenfehler; beide waren mit Erfolg mit Chinidin behandelt worden.
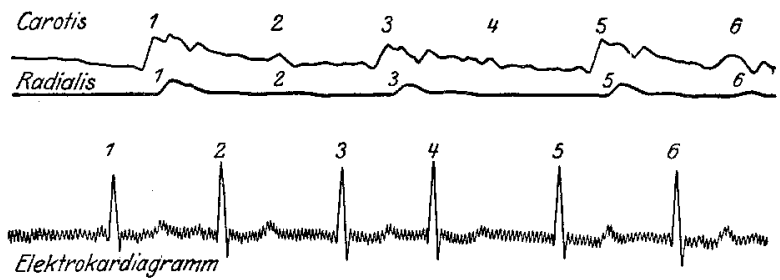

Abb. I. Flektrokardiogramm (unten) nebst Carotis- und Radialispulskurven (oben) eines 22 jähr. Mannes (Tab. I, Nr. 26) mit Arbythmia perpetua. Man sieht den Puls sich gegen die Peripherie hin verlieren, so da $B$ in der Radialis weniger $\mathbf{P u l s s c h l a ̈ g e ~ v o r k o m m e n ~}$ als in der Carotis.

Das Pulsdefizit und sein Zunehmen, allmählich wie man sich der Peripherie nähert, ist in Fig. I direkt veranschaulicht. Auf derselben photographischen Platte ist ein Elektrokardiogramm und 2 Arterienpulskurven, eine von der Carotis und eine von der Radialis, aufgenommen $\mathbf{1}$ ). An dem Elektrokardiogramm sieht man 6 R-Gipfel, welche den Anfang der entsprechenden Ventrikelkontraktionen kennzeichnen. Sie haben keinen Vorkammergipfel (P-Gipfel) vor sich und sind durch unregelmäßige $Z$ wischenräume getrennt, wie es bei Atriumflimmern und Arhythmia perpetua vorkommt. An der Carotiskurve lassen sich alle 6 Herzschläge erkennen, wenn 2 von ihnen auch sehr klein sind und einer fast verschwunden ist. Auf dem Wege nach dem Handgelenk hinab ist der kleinste der Carotisgipfel (Nr.4) ganz verschwunden, so daB er sich an der Radialiskurve nicht zu erkennen gibt. Ein anderer der Radialisgipfel (Nr. 2) ist eben sichtbar und wird bei Palpation nicht deutlich zu erkennen sein. Übrig sind nur 4 deutliche Pulsschläge am Handgelenk, so daß wir ein Defizit

i) Zur hier angewandten Technik siehe C. LUNDSGAARD und O. BEYERHOLM: En ny Metode $t$ t! Maaling af Pulsboelgens Hastighed. Bibliothek for Laeger, Maj $192 x$ und Nouvelle methode pour mesurer la vitesse.de propagation de l'onde pulsat. arterielle. Compte rend. Soc. Biol, 85, 371. I921. 
von 2 oder $33 \%$ erhalten. Die Kurve gewährt einen unmittelbaren Eindruck davon, daß eine solche Herztätigkeit dem Vortreiben des Blutes nicht förderlich sein kann.

\section{Die Bedeutung des Pulsdefizits für die Diagnose der} Arhythmia perpetua (Differentialdiagnose).

Eine Arhythmia mit einem ausgesprochenen Pulsdefizit wird in der Regel eine Arhythmia perpetua sein; man kann aber doch nicht das Vorhandensein eines Pulsdefizites als pathognomionisch für diese Arhythmieform betrachten. Ein Pulsdefizit kommt auch bei Extrasystolie vor, wenn auch nur ausnahmsweise in so hohem Grade wie bei Arhythmia perpetua ${ }^{1}$ ). In der Regel wird man jedoch leicht entscheiden können, ob man mit der einen oder der anderen dieser wichtigen und häufig vorkommenden Arhythmieformen zu tun hat.

Erstens kennzeichnet die Extrasystolie sich dadurch, daß Perioden mit vollständig regelmäßigem Rhythmus durch mehr oder minder periodisch wiederkehrende Pulspausen abgelöst werden. Diese entsprechen den extrasystolischen (prämaturen) Herzkontraktionen, die nicht vermocht haben, ein hinreichend großes Schlagvolumen auszusenden, um eine am Handgelenk palpierbare Pulswelle zu ergeben. Dementsprechend wird man, jedenfalls was die ventrikulären Extrasystolen betrifft, den Rhythmus wieder aufnehmen können, wenn man, ungeachtet der ausfallenden Schläge, in dem einmal getroffenen Takt weiterzählt. Im Gegensatz dazu findet sich bei Arhythmia perpetua ein vollkommener Mangel an Rhythmus.

Ein zweites wichtiges differentialdiagnostisches Kennzeichen liegt in dem Umstand, daß man bei Extrasystolie mit Pulsdefizit in den allermeisten Fällen eine Herzfrequenz vorfinden wird, die entweder normal oder langsamer als normal ist, während bei Arhythmia perpetua in der Regel das Entgegengesetzte der Fall sein wird (siehe Tab. I). Man kann sagen, da $B$ ein Pulsdefizit sich bei der Extrasystolie als eine Bradysphygmie mit normaler Herzfrequenz (Normocardie) zu erkennen gibt, während dasselbe sich bei der Arhythmia perpetua durch eine Tachycardie mit Normosphygmie oder mäßiger Tachysphygmie ausspricht.

SchlieBlich hat man als drittes differentialdiagnostisches Kennzeichen das Verhalten des Pulsdefizits bei der Ausführung einer gewissen Arbeit. Die Hauptregel ist, daß gleich nach Anstrengungen (Widerstandsbewegungen, gewöhnlichem Gang oder Treppensteigen) ein bei Patienten mit Extrasystolie bei Ruhe nachgewiesenes Pulsdefizit sich in den allermeisten Fällen entweder vermindern oder ganz aufhören wird, während cs sich bei Patienten mit Arhythmia perpetua entweder steigert (meist sehr bedeutend) oder, wenn es bei Ruhe nicht vorlag, sich hervortut. Jedoch ist diese Regel nicht ohne Ausnahme. Nicht so sehr selten. sieht man Extrasystolen wegen Anstrengungen entstehen, was namentlich bei digitalisbehandelten Patienten der Fall ist, $u$. a. nicht selten bei Patienten mit Arhythmia perpetua. Die Ursache liegt zuvörderst in der Neigung der Digitalis, bei längerer Verabreichung und in großen Gaben Extrasystolen hervorzurufen; jedoch tragen die Myocardveränderungen sicherlich auch etwas von der Schuld. Andererseits können kräftig mit Digitalis behandelte Patienten mit Arhythmia perpetua mitunter auch nicht ganz geringe Anstrengungen aushalten, ohne daß ein Pulsdefizit eintritt (siehe z. B. Tab. IV Nr. I). SchlieBlich sieht man ausnahmsweise auch ein Pulsdefizit bei Arhythmia perpetua nach (leichteren) Anstrengungen abnehmen, was sich jedoch aus der großen Variabilität der Herztätigkeit bei diesen Patienten erklären läßt. Auch sieht man oft ein Pulsdefizit von Minute zu Minute bedeutend an Größe schwanken.

Tab. II enthält einige Beispiele von dem Verhalten des Pulsdetizites nach Anstrengungen. Die Herz- und Pulsfrequenz wurde gezählt, sowohl während die Patienten sich ruhig verhielten als nach Ausführung einer gewissen . Arbeit;

1) Mitunter kann man jeđoch bei der Extrasystolie ein sehr großes Pulsdefizit vorfinden nämlich wenn jeder zweite oder jeder dritte Schlag eine Extrasystole darstellt, die das Handgelenk nicht erreicht. Die Differentialdiagnose wird sich in einem solchen Falle zwischen partiellem Herzblock und Extrasystolie bewegen. - Auch bei der paroxysmatischen Tachycardie kann mitunter ein Pulsdefizit vorkommen. Die klinische Differentialdiagnose dieser Leiden soll hier nicht mehr behandelt werden.
Die beiden Untersuchungen fanden unmittelbar nacheinander statt. Bei dem einen Patienten ergab sich bei Ruhe kein Pulsdefizit. Das Treppensteigen bewirkte ein Pulsdefizit von 44 . Bei den übrigen 7 Patienten steigerte sich ein bereits bei Ruhe vorliegendes Defizit. Man sieht zugleich, da $B$ die Bewegung in allen Fällen eine bedeutende Tachykardie hervorruft, während die Häufigkeit des Radialispulses sich nur sehr wenig steigert, in einigen Fällen - Nr. I, 4 und 9 - sieht man sie sogar abnehmen.

Tabelle II.

Tabelle zur Veranschaulichung des Einflusses von Anstrengungen auf das Pulsdefizit bei Patienten mit Arhythmia perpetua.

\begin{tabular}{|c|c|c|c|c|c|c|c|c|}
\hline \multirow{2}{*}{\multicolumn{2}{|c|}{ 党| }} & \multicolumn{2}{|c|}{ Ruhe } & \multicolumn{2}{|c|}{ Bewegung } & \multicolumn{2}{|c|}{ Pulsdefizit } & \multirow[b]{2}{*}{ Anmerkung } \\
\hline & & 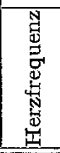 & 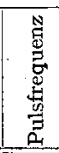 & 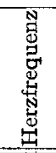 & 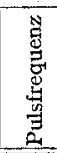 & 营 & 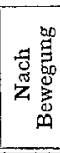 & \\
\hline $\mathrm{I}$ & 9 & 88 & 88 & 128 & 84 & o & 44 & Treppe bis zum I. Stock I mal. \\
\hline 2 & 4 & 80 & 78 & 156 & II 2 & 2 & 44 & Treppe bis zum I. Stock 5 mal. \\
\hline 3 & 7 & IIO & 102 & 158 & 100 & 8 & 58 & Treppe bis zum I. Stock I mal. \\
\hline 4 & 12 & 80 & 76 & 180 & 58 & 4 & 122 & \\
\hline 5 & 6 & 84 & 82 & 190 & $\mathrm{I} 42$ & 2 & 48 & \\
\hline 6 & IO & 122 & 66 & 142 & 76 & 56 & 66 & im Zimmer gehend. \\
\hline 7 & I I & 140 & 108 & 152 & II 2 & 32 & 40 & \\
\hline 8 & 8 & 90 & 78 & 126 & 82 & I 2 & 44 & \\
\hline 9 & 13 & I 34 & 80 & I 76 & 74 & 54 & 102 & \\
\hline
\end{tabular}

Die Bedeutung des Pulsdefizits für die Einschätzung des funktionellen Zustandes des Herzens (Funktions-Diagnose).

Wie bekannt, ist namentlich von WENCKEBACH, J. MACKENzIE und TH. LewIs behauptet worden, daß dic Arbeitsweise des Herzens von ebenso großer Bedeutung für den Blutumlauf ist wie die Arbeitskraft. Diese Ansicht, die im wesentlichen auf klinischen Beobachtungen und Erörterungen begründet wurde, und die besagt, daß eine unregelmäßige Herztätigkeit an und für sich eine Herabsetzung der von dem Herzen in der Zeiteinheit ausgesandten Blutmenge bewirken kann, ist durch direktes Messen des Minutvolums des Herzens (C. LUNDSGAARD) durch Messen der Kohlensäurespannung des Venen- und Arterienblutes (SONNE) und des Sauerstoffgehaltes des Venenblutes (C. LUNDSGAARD) experimentell bestätigt worden. In allerneuster Zeit zugleich durch gleichzeitiges Messen der Sauerstoffmenge des Arterien- und Venenblutes (STADIE, Harrop). Diese verschiedenen Untersuchungen ergaben alle übereinstimmende Resultate, obschon die in den einzelnen Fällen benutzte Untersuchungsmethode verschieden war. Dazu kommen noch aus der allerjüngsten Zeit verschiedene durch die Chinidintherapie gewonnenen Erfahrungen. Falls das Chinidin auf einen Patienten mit Arhythmia perpetua wirkt, sieht man oft den Puls auf einmal langsamer und vollständig regelmäßig werden (Abb. 5). Gleichzeitig stellt sich eine augenblickliche sowohl subjektiv als objektiv sehr ausgesprochene Besserung des Zustandes des Patienten ein. Diese Beobachtungen entsprechen ganz demjenigen, was $z$. B. MackenzIE bei Patienten wahrgenommen hat, die von einer Arhythmia perpetua betroffen wurden; sie können jedoch wegen ihres experimentellen Charakters weit mehr verblüffend auf den Beobachter wirken. Es kann somit als klinisch und experimentell dargetan betrachtet werden, daß eine unregelmäßige Herzaktion eine für das Herz unzweckmäßige Arbeitsweise darstellt, indem sie eine Herabsetzung der in der Zeiteinheit ausgesandten Blutmenge bewirkt. Im Vorhergehenden ist besprochen worden, daß man oft ein Pulsdefizit hervorrufen bzw. steigern kann, wenn man den Patienten eine gewisse Arbeit ausführen läßt. Eine nähere Untersuchung dieses Verhältnisses zeigt, daß ein Pulsdefizit im großen ganzen mit der Größe der Arbeitsleistung gleichmäßig zunimmt. Die Tab. III und VI enthalten ein paar Beispiele davon. Man sieht ein starkes Zunehmen der Herzfrequenz, während die Pulshäufigkeit entweder sehr wenig zunimmt oder (seitener) abnimmt. Bei dem einen Patienten (E. N.) beträgt die Pulsfrequenz nach dem Gehen im Zimmer Ir2, nach dem Treppensteigen aber nur Io8; die entsprechenden Zahlen 
Tabelle III.

Das Verhalten des Pulsdefizits während zunehmender Arbeitsleistung bei zwei Patienten mit Arhythmia perpetua. Die Zählungen fanden unmittelbar nacheinander statt, sobald Patient ausgeruht hatte. - Vgl. Tabelle II u. VI.

\begin{tabular}{|c|c|c|c|c|c|}
\hline & & 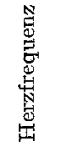 & 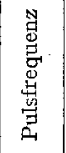 & 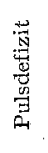 & Anmerkungen \\
\hline \multirow{5}{*}{$\begin{array}{c}\text { Nr. I } \\
\text { (Nr. I } 3 \\
\text { in Tab. I) }\end{array}$} & E. N. 오 & $\mathrm{I} 26$ & 94 & 32 & Ruhe \\
\hline & 37 Jahre & I 40 & 96 & 44 & $\begin{array}{l}5 \text { maliges Armstrecken gegen } \\
\text { Widerstand }\end{array}$ \\
\hline & & I 74 & II 2 & 62 & $\begin{array}{l}\text { etwa } 1 / 2 \text { Min. langes Gehen } \\
\text { im Zimmer }\end{array}$ \\
\hline & $16 / 921$ & I 76 & 108 & 68 & Treppe bis zum I. Stock Imal. \\
\hline & V.E.N.오 & I 20 & 82 & 38 & Ruhe \\
\hline $\begin{array}{l}\text { Nr. } 2 \\
\text { (Nr. I7 }\end{array}$ & 25 Jahre & I 36 & 86 & 50 & $\begin{array}{l}5 \text { maliges Armstrecken gegen } \\
\text { Widerstand }\end{array}$ \\
\hline in Tab. I) & $14 / 921$ & 170 & 80 & 90 & Gehen im Zimmer \\
\hline
\end{tabular}

der Herzfrequenz sind sich fast gleich, nämlich $\mathrm{I} 74$ und $\mathrm{I} 76$. Bei dem anderen Patienten (V. E. N.) sieht man auch die
Pulsdefizits gewährt uns daher einen gewissen Eindruck von dem vorliegenden funktionellen Zustande des Kreislaufes. Solange es sich um einen Patienten handelt, dessen Kreislauf ",absolut insuffizient" ist, besitzen wir allerdings an der Dyspnöe, Cyanose, Stasis usw. Symptome genug, um den Zustand beurteilen zu können, so daß ein Messen des Pulsdefizites zu diesem Zeitpunkt mehr Bedeutung für die Differentialdiagnose erhält als für die Funktionsdiagnose.

Das Verhältnis zwischen der absoluten und der relativen Herzinsuffizienz ist bei weitem noch nicht aufgeklärt, es läßt sich jedoch einsehen, daß mehrere tiefgreifende und wichtige Unterschiede bestehen. Die absolute Insuffizienz ist aktuell und kontinuierlich, die relative aber potentiell und nurwährend der Zeit wirksam, in welcher an das Herz gesteigerte Ansprüche gestellt werden. Wenn eine absolute Herzinsuffizienz längere Zeit hindurch bestanden hat, werden in der Regel irreparable organische Veränderungen (Cirrhose cardiaque, braune Lungeninduration usw.) entstanden sein, die gewissermaßen als neue selbstständige Leiden wirken. Die absolute bewirkt einen unhinlänglichen Transport von sowohl Sauerstoff als Flüssigkeit. Im Gegensatz dazu stellt die relative hauptsächlich nur eine Sauerstoffinsuffizienz dar, d. h. einen im Verhältnis zu den gesteigerten Ansprüchen unhinlänglichen

Tabelle IV.

Ubersicht über das Verhalten des Pulsdefizits zu verschiedenen Zeitpunkten während des Krankenhausaufenthaltes einiger Patienten. Was den funktionellen Zustand des Kreislaufes der Patienten betrifft, wird auf den Text verwiesen.

\begin{tabular}{|c|c|c|c|c|c|c|c|c|c|c|c|c|}
\hline \multirow[t]{2}{*}{ Nr. } & \multirow{2}{*}{$\begin{array}{l}\text { Name u. Nr. } \\
\text { von Tab. I }\end{array}$} & \multirow{2}{*}{$\begin{array}{c}\text { Datum } \\
\text { der } \\
\text { Unter- } \\
\text { suchung }\end{array}$} & \multicolumn{3}{|c|}{ Ruhe } & \multicolumn{3}{|c|}{ Gehen im Zimmer } & \multicolumn{3}{|c|}{$\begin{array}{c}\text { Treppensteigen } \\
\text { Parterre } \\
\text { bis zum I. Stock }\end{array}$} & \multirow[t]{2}{*}{ Anmarkungen } \\
\hline & & & Apex & Puls & Defizit & Apex & Puls & Defizit & Apex & Puls & Defizit & \\
\hline I & $\begin{array}{l}\text { S. C. } \\
\text { Nr. II }\end{array}$ & $\begin{array}{l}15 / 8 \\
20 \% / 8 \\
25 / 9\end{array}$ & $\begin{array}{r}140 \\
92 \\
76\end{array}$ & $\begin{array}{r}108 \\
92 \\
76\end{array}$ & $\begin{array}{r}32 \\
0 \\
0\end{array}$ & $\begin{array}{l}152 \\
108 \\
-\end{array}$ & $\begin{array}{r}\text { I } 2 \\
98 \\
-\end{array}$ & $\begin{array}{l}40 \\
10 \\
-\end{array}$ & $\begin{array}{l}-\overline{118} \\
100\end{array}$ & $\begin{array}{l}- \\
104 \\
100\end{array}$ & $\begin{array}{r}- \\
4 \\
0\end{array}$ & I maliges Treppensteigen \\
\hline 2 & $\begin{array}{l}\text { C. V. O. } \\
\text { Nr. } 9\end{array}$ & $\begin{array}{l}28 / 7 \\
16 / 9\end{array}$ & $\begin{array}{r}\text { II } 8 \\
88\end{array}$ & $\begin{array}{l}64 \\
88\end{array}$ & $\begin{array}{r}54 \\
0\end{array}$ & $\frac{\mathrm{I}_{4} 2}{-}$ & $\frac{78}{-}$ & 64 & $\begin{array}{l}180 \\
128\end{array}$ & $\begin{array}{r}I 00 \\
84\end{array}$ & $\begin{array}{l}80 \\
44 \\
\end{array}$ & I maliges Treppensteigen \\
\hline 3 & $\begin{array}{l}\text { O. B. } \\
\text { Nr. } 7\end{array}$ & $\begin{array}{l}22 / 4 \\
29 / 4\end{array}$ & $\begin{array}{r}\text { I } 30 \\
74 \\
\end{array}$ & $\begin{array}{l}88 \\
72 \\
\end{array}$ & $\begin{array}{r}42 \\
2\end{array}$ & 二 & - & - & $\begin{array}{l}\text { I72 } \\
\text { IOO }\end{array}$ & $\begin{array}{r}104 \\
90 \\
\end{array}$ & $\begin{array}{l}68 \\
10\end{array}$ & 2 maliges Treppensteigen \\
\hline 4 & $\begin{array}{l}\text { J. P. H. } \\
\text { Nr. } 4\end{array}$ & $\begin{array}{l}28 / 1 \\
15 / 2 \\
\end{array}$ & $\begin{array}{r}\text { IO4 } \\
80\end{array}$ & $\begin{array}{l}76 \\
78\end{array}$ & $\begin{array}{r}28 \\
2\end{array}$ & I 36 & $\stackrel{88}{-}$ & 48 & - & $\overline{\mathrm{II} 2}$ & - & 5 maliges Treppensteigen \\
\hline 5 & $\begin{array}{l}\text { C. H. } \\
\text { Nr. } 16\end{array}$ & $\begin{array}{r}15 / 8 \\
6 \% 9 \\
17 / 9\end{array}$ & $\begin{array}{r}\text { I } 30 \\
64 \\
72\end{array}$ & $\begin{array}{l}80 \\
64 \\
72\end{array}$ & $\begin{array}{r}50 \\
0 \\
0\end{array}$ & $\overline{80}$ & $\begin{array}{l}-\overline{80} \\
-\end{array}$ & $\begin{array}{l}- \\
-\end{array}$ & $\overline{-}$ & $\frac{\bar{Z}}{104}$ & $\frac{-}{0}$ & I maliges Treppensteigen \\
\hline
\end{tabular}

Pulsfrequenz nach einem Gehen in der Stube ein wenig abnehmen, indem sie nach einer Ausführung von Widerstandsbewegungen mit den Armen 86, nach einem Gehen aber nur 80 beträgt. Daß das Gehen an die Zirkulation größere Ansprüche gestellt hat als die Armbewegungen, kann als gegeben betrachtet werden, geht im übrigen aber aus dem Umstand hervor, daß die Herzfrequenz von 136 auf 17 o steigt. Es ist unmittelbar einleuchtend, daß die Bedingungen für eine gesteigerte Zirkulation während der Arbeit unter solchen Verhältnissen ungünstig sind ${ }^{1}$ ).

Wir besitzen bei weitem nicht klinische und experimentelle Beobachtungen genug, um entscheiden zu können, inwiefern und in welchem Verhältnis đie Kreislaufinsuffizienz mit dem Grade des Pulsdefizits zunimmt. A priori ist ein Parallelismus zwischen den beiden natürlicherweise unwahrscheinlich. So viel wissen wir jedoch aus klinischen Beobachtungen, daß eine sehr schnelle und unregelmäßige Herzaktion mit hohem Defizit oft mit ausgesprochenen Insuffizienzsymptomen verbunden ist, während eine unregelmäBige Herztätigkeit, die langsam ist und kein Pulsdefizit aufweist, keine Insuffizienzsymptome zu ergeben braucht, jedenfalls nicht, wenn der Patient sich ruhig verhält. Ein Feststellen der Größe des

${ }_{\text {I }}$ Es ist in dieser Verbindung von Interesse, des früher erwähnten Verbältnisses zu gedenken, dab Extrasystolen meist schwinden, wen das Individuum, sich beivegt, da dies als eine der Ursachen dazu bettachtet werden $m u{ }^{\prime}$, dab die Extrasystolie ein auch für die augenblickliche Funktion weit unschuldigeres Leiden darstellt als die Arhythmia perpetia.
Transport von sauerstofftragenden Teilen (vgl. das Verhältnis bei der Anämie). Dem entsprechen die klinischen Symptome: bei der absoluten Insuffizienz sind sie vielgestaltig und rühren nicht nur von einer ungenügenden Sauerstoffzufuhr (Dyspnöe, Cyanose), sondern auch von einer abnormen Flüssigkeitsverteilung und einem abnormen Flüssigkeitstransport (Stasis, Odeme, verminderte Diurese) her. Bei der relativen besteht nur Dyspnöe und Cyanose. Indessen können die Dyspnöe und Cyanose auch von anderen Ursachen als Leiden des Herzens herrühren; so können Lungenleiden oft diese beiden Symptome ergeben, wie bei der Anämie eine ausgesprochene Arbeitsdyspnöe vorliegen kann. Eine Untersuchung des Verhaltens des Pulsdefizites bei Arbeit hat daher Bedeutung als Hilfsmittel für die Entscheidung, inwiefern eine relative kardiale Kreislaufinsuffizienz vorliegt.

Wir untersuchten bei den meisten unserer Patienten mit gewissen Zwischenräumen, oft täglich, das Pulsdefizit sowohl nach Ruhe als nach abgeänderten Anstrengungen. Es ergab sich im großen ganzen, daß eine Abnahme der Größe des Pulsdefizites einer Besserung des subjektiven und objektiven Zustandes des Patienten entspricht. Tab. IV enthält einige Beispiele von solchen Untersuchungen an verschiedenen Patienten.

Für Pat. I enthält die Tabelle 3 Zählungen aus 3 verschiedenen Perioden des Krankenhausaufenthaltes. Bei der Aufnahme bestand ein sehr bedeutendes Pulsdefizit sowohl in Ruhe wie nach 
dem Gehen in der Stube. Es lag zu diesem Zeitpunkt ausgesprochene absolute Insuffizienz des Herzens vor. Nach einiger Behandlung und zu einem Zeitpunkt, wo die Symptome der absoluten Insuffizienz geschwunden waren, ergab sich bei Ruhe kein Pulsdefizit. Nach einem Gehen in der Stube ein Defizit von Io und nach dem Treppensteigen I4. Gleichzeitig hatte die Herzaktion von 92 bei Ruhe bis i 8 nach Treppensteigen zugenommen. Klinisch lag zu diesem Zeitpunkt Arbeitsdyspnơe als Anzeichen von relativer In suffizienz vor. Zu Ende des Krankenhausaufenthaltes konnte Pat. recht schnell die Treppe bis zum ersten Stockwerk eines Pavillons hinaufsteigen, ohne daß ein Defizit eintrat, und ertrug es jetzt besser als früher und wies nur geringe Arbeitsdyspnöe auf.

Pat. 2 (C. V. O.) wies bei der ersten Zählung gleichfalls Anzeichen absoluter Insuffizienz auf. Gleichzeitig wurde sowohl bei Ruhe als nach Arbeit ein deutliches Defizit nachgewiesen. Zu Ende der Behandlung lag bei Ruhe kein Pulsdefizit vor, and die Frequenz war nur wenig beschleunigt. Nach Treppensteigen stieg die Herzfrequenz recht stark, und gleichzeitig nahm die Pulsfrequenz ab, so daß sich ein bedeutendes Defizit ergab. Die Zirkulation dieses Patienten schien eine gute zu sein, wenn man ihn nur bei Ruhe betrachtete, er ertrug Anstrengungen aber sehr schlecht. Das Verhalten des Pulses macht dies leicht verständlich. Die Reservekraft seines Herzens war entweder gering oder konnte wegen der bei Anstrengungen eintretenden schlechten Bedingungen für das Vortreiben des Blutes nicht effektiv werden.

Pat. 3 (O. B.) wies bei der Aufnahme, als die erste Zählung stattfand, keine Anzeichen von absoluter Herzinsuffizienz. Nichtsdestoweniger finden wir einen recht schnellen Puls ( I 30 ) und ein bedeutendes Defizit bei Ruhe. Nach Treppensteigen, das ausgesprochene Funktionsdyspnöe ergab, nahm das Pulsdefizit kräftig zu. $Z u$ Ende der Behandlung fand sich ein Defizit von 2, was ohne Bedeutung ist. Nach Treppensteigen stieg es bis auf ro, so daß die Zirkulation nicht als bei Anstrengungen genügend betrachtet werden kann.

Pat. 4 (J.P. H.) wies dasselbe Verhältnis auf, wenn auch in weit ausgesprochenerem Grade. Bei der ersten Zählung befanden wir uns an der Grenze der absoluten Insuffizienz, bei der zweiten lagen nur Symptome relativer Insuffizienz vor. Pat. war Landpostbote und hatte immer lange Strecken zu gehen oder zu radeln, oft mit einer nicht geringen Last beladen. Er ertrug diese Arbeit sehr schlecht. Dies erklärt sich aus dem Verhalten des Pulsdefizites nach Treppensteigen. Auch nach einem langen Aufenthalt im Krankenhaus lag noch deutliche relative Insuffizienz vor.

Pat. 5 (C. H.) wies bei der Aufnahme schon bei Ruhe ein bedeutendes Defizit auf. Gleichzeitig Iagen deutliche Insuffizienzsymptome vor. Nach einer Chinidinbehandlung schwanden die Symptome der absoluten Insuffizienz schnell. Gleichzeitig wurde der Puls regelmäßig sowohl bei Ruhe als nach Anstrengungen. Trotzdem bestanden noch fortwährend Symptome von relativer Insuffizienz, indem Pat. bei Treppensteigen (leicht) dyspnöisch wurde. Die Ursache der relativen Insuffizienz ist annehmbar in einer Schwächung des Myokardiums, vielleicht auch in chronischen Veränderungen in den Lungen zu suchen. Pat. wurde mit Symptomen von Lungenödem im Krankenhaus aufgenommen.

Die Bedeutung des Pulsdefizits für die Therapie.

Es ist immer als wichtig betrachtet worden, bei der Behandlung von Patienten mit Herzleiden die Häufigkeit des Pulses zu kontrollieren. Namentlich ist auf ein tägliches Zählen des Pulses während einer Digitaliskur Gewicht gelegt worden. Gleichfalls betrachtet man in der Regel eine Behandlung mit Kohlensäurebädern als effektiv, wenn man eine (geringere) Abnahme der Pulsfrequenz nach einem solchen Bade feststellen kann. Die in dieser Arbeit dargestellten Beispiele vom Unterschied zwischen der Häufigkeit des Herzens und des Pulses bei Patienten mit Arhythmia perpetua sind sicherlich hinreichend überzeugende Belege davon, daß ein isoliertes Pulszählen fast nichts zu besagen braucht. Es kommt nicht selten vor, daß man die-Pulsfrequenz während des Anfangs einer Digitalisbehandlung bei einem Patienten mit Arhythmia perpetua steigen sieht. Diese Erscheinung bedeutet nicht eine mangelhafte, geschweige denn eine schädliche Wirkung der Behandlung, im Gegenteil: sie bedeutet nur, daß eine größere Anzahl der Herzschläge wegen eines besseren Füllens oder einer besseren. Kontraktion der Ventrikel bis an das. Handgelenk, ,gelangen". Etwas besser als ein Pulszählen wäre eine Kontrolle der Frequenz des Herzens; am besten beobachtet man aber sowohl đie Frequenz des Herzens als die Häufigkeit des Pulses und berechnet daraus das Pulsdefizit.
Bei der Behandlung von Patienten mit Arhythmia perpetua muß man darauf abzielen, ein etwa vorliegendes Pulsdefizit zum Schwinden zu bringen. Dadurch hebt man in der Regel zugleich die Tachykardie auf und verschafft der Zirkulation bessere Bedingungen. Sofern der Patient sich in einem Stadium von absoluter Herzinsuffizienz befindet, werden, wie früher erwähnt, eine Reihe von anderen Symptomen als das Pulsdefizit vorliegen, die für unser therapeutisches Verfahren eine Richtschnur abgeben können. Befindet der Patient sich dagegen in einem Stadium von relativer Insuffizienz, wird das Pulsdefizit von bedeutendem Wert sein sowohl für die Wahl der Arznei, als für die Entscheidung der.Frage, wie lange und wie intensiv man behandeln soll. Ferner kann das Verhalten des Pulsdefizites für die Entscheidung der Frage, wie der Patient in der Zukunft seine Lebensführung regeln soll, maßgebend sein.

Was die erste Frage betrifft, wird eine fortwährende Kontrolle des Pulsdefizits (evtl. nach Arbeit) uns ab und $z u$ darüber aufklären können, daß das Präparat (z. B. das Digitalispräparat), das wir anwenden, nicht die erwünschte Wirkung hat. Sofern der Grund nicht darin liegt, daß die Größe der Gabe nicht die angemessene (gewöhnlich zu klein) ist, kann man mitunter durch ein anderes Präparat einen guten Erfolg erzielen. Mitunter ist es am besten, Digitalispräparate zu wechseln, während es in anderen Fällen am erfolgreichsten ist, zu Strophantus überzugehen. Was die zweite Frage, die Dauer und Intensität der Behandlung, betrifft, hat man möglichst die Therapie so lange fortzusetzen, bis der Patient imstande ist, eine gewisse Anstrengung zu ertragen, z. B. eine Treppe steigen kann, ohne daß sich dadurch ein Pulsdefizit einstellt. Sich hier allein nach dem Verhalten des Pulsdefizits bei Ruhe zu richten, wäre, wie aus Tab. IV und den folgenden Tabellen hervorgeht, unberechtigt.

SchlieBlich muß man bei der Nachuntersuchung von Patienten mit Arhythmia perpetua ihr Pulsdefizit nach einer ihren Verhältnissen angemessenen Anstrengung untersuchen, damit man bei Zeiten mit der Behandlung eingreifen kann. So wird man möglicherweise das Hinübergleiten in ein Stadium absoluter Insuffizienz verzögern können. Bei verschiedenen

Tabelle V.

Resultate von Zählungen der Herzfrequenz und Pulshäufigkeit bei Ruhe und nach Anstrengungen bei einem Patienten mit Arhythmia perpetua. Insufficientia und Stenosis mitralis. (Vgl. Abb. 3, sowie Tabelle I, Nr. I I u. Tabelle IV, Nr. I.)

\begin{tabular}{|c|c|c|c|c|c|}
\hline Datum & Bedingungen & 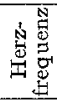 & 造 & $\begin{array}{l}\text { 苫 } \\
\text { 荧 } \\
\text { A }\end{array}$ & Anmerkungen \\
\hline $13 / 8$ I 9 & Ruhe & I 72 & 92 & 82 & \multirow{4}{*}{ keine Herztonica } \\
\hline $14 / 819$ & Ruhe & $\mathrm{I}_{42}$ & 104 & 38 & \\
\hline \multirow{2}{*}{$15 / 8$ I9 } & Ruhe & $x_{40}$ & 108 & 32 & \\
\hline & Gehen im Zimmer & $x_{5}^{2}$ & $\mathrm{x} \pm 2$ & 40 & \\
\hline \multirow{2}{*}{$16 / 819$} & Ruhe & 140 & I I 6 & 24 & \multirow{8}{*}{$\begin{array}{c}3 \text { mal I } 5 \text { Tropfen } \\
\text { Digalen }\end{array}$} \\
\hline & Gehen & 150 & 126 & 24 & \\
\hline \multirow{2}{*}{$18 / 819$} & Ruhe & I 36 & $I_{4}$ & 22 & \\
\hline & Gehen & $\mathrm{I}_{42} 2$ & I I 2 & 30 & \\
\hline \multirow{3}{*}{$19 / 819$} & Ruhe & I 24 & I I 4 & Io & \\
\hline & Gehen & $\mathrm{T} 24$ & II 8 & 6 & \\
\hline & Ruhe & 92 & 92 & 0 & \\
\hline $2.3 / 819$ & $\begin{array}{l}\text { Gehen - } \\
\text { Treppensteigen }\end{array}$ & $\begin{array}{l}108 \\
118\end{array}$ & $\begin{array}{r}98 \\
\text { T.04 }\end{array}$ & IO & \\
\hline \multirow{3}{*}{$21 / 819$} & Ruhe & 92 & 92 & $\begin{array}{r}14 \\
0\end{array}$ & \\
\hline & Gehen & 94 & 92 & 2 & \\
\hline & Treppensteigen & rio & 94 & 16 & \\
\hline \multirow{2}{*}{$22 / 819$} & Ruhe & 88 & 88 & 0 & \\
\hline & Treppensteigen & 106 & $\mathrm{IO} 2$ & 4 & \\
\hline \multirow{2}{*}{$25 / 8$ I9 } & Ruhe & 80 & 80 & o & \\
\hline & Treppensteigen & 94 & 94 & 0 & 3 mal io Tropfen \\
\hline $28 / 819$ & Ruhe & 80 & 80 & 0 & Digalen \\
\hline \multirow{2}{*}{$2 / 9+9$} & $\begin{array}{l}\text { 1reppensteigen } \\
\text { Ruhe }\end{array}$ & $\begin{array}{r}100 \\
80\end{array}$ & $\begin{array}{r}106 \\
80\end{array}$ & $\begin{array}{l}0 \\
0\end{array}$ & $3 \mathrm{mal}=$ Tropfen \\
\hline & Treppensteigen & I I 4 & 114 & $\mathrm{O}$ & Digalen \\
\hline \multirow{2}{*}{$16 / 919$} & Ruhe & 80 & 80 & $\mathrm{O}$ & . \\
\hline & Treppensteigen & 98 & 98 & O & 1 mal 5 Tropfen \\
\hline \multirow[t]{2}{*}{$25 / 9$ I9 } & $\begin{array}{l}\text { Ruhe } \\
\text { Treppensteigen }\end{array}$ & $\begin{array}{r}76 \\
100\end{array}$ & $\begin{array}{r}76 \\
100\end{array}$ & $\begin{array}{l}0 \\
0\end{array}$ & Digalen \\
\hline & & & & & \\
\hline
\end{tabular}


Patienten mit Arhythmia perpetua wird man es nicht so weit bringen können, daß sie auch nur leichtere Anstrengungen ertragen, ohne daß sich ein Pulsdefizit einstellt, und etliche werden sogar trotz sorgfältiger und langwieriger Behandlung bei Ruhe ein Defizit aufweisen.

In sämtlichen Fällen muß man das Verhalten des Pulsdefizites (nach Ruhe oder nach angemessenen Anstrengungen) bei der Regelung der Lebensführung der Patienten nach dem Aufhören der eigentlichen Kur mit in Betracht ziehen.

Die Tab. V und die Abb. 3-6 enthalten Beispiele von dem Verhalten des Pulsdefizites während des Krankenhausaufenthaltes verschiedener Patienten. Tab. V rührt von einem Pat mit Insufficientia et Stenosis mitralis her (Tab. I, Nr. II). Die Patientin findet sich auch in Tab. IV (Nr. I), und im Text finden sich, wo diese Tabelle besprochen wird, einige Mitteilungen.

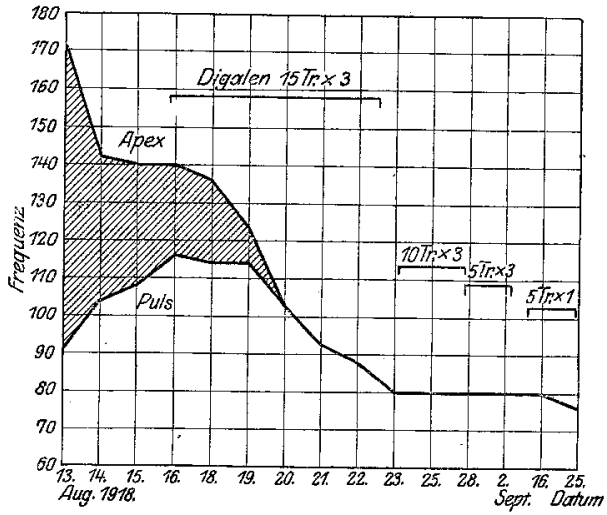

Abb. 2. Graphische Darstellung des Verhaltens des Pulsdefizits bei einem Patienten (Tab. I, Nr. II) während einer Digitaliskur.

In Tab.V findet sich das Verhalten des Pulsdefizites sowohl bei Ruhe als nach Bewegung während des Krankenhausaufenthaltes angegeben. Abb. 2 enthält Kurven über die Herzfrequenz (oben)

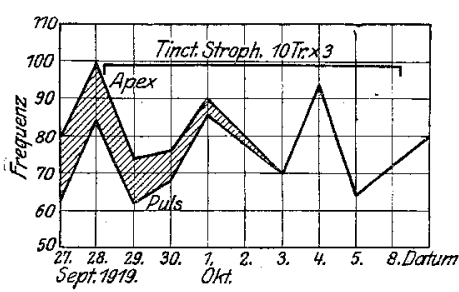

Abb. 3. Graphische Darstellung des Verhaltens des Pulsdefizits bei einem Patienten (Tab. I, Nr. 4) während einer Strophantuskur. fortwährend vermindert, die Herzaktion wird langsamer, ist aber unregelmäßig, indem das Atriumflimmern, das die Arhythmie bewirkt, sich unverändert erhält.

Tabelle VI.

Resultate von Zählungen der Herzfrequenz und Pulsfrequenz bei Ruhe und nach schwankenden Anstrengungen bei einem 48jährigen Mann mit Arhythmia perpetua und Stenosis mitralis. (Vgl. Tab. I, Nr. 4 und $\mathrm{Abb}$. 3.)

\begin{tabular}{|c|c|c|c|c|c|}
\hline Datum & Bedingungen & 怘兽 & 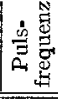 & $\begin{array}{l}\stackrel{\vec{N}}{\mathbb{N}} \\
\stackrel{\mathbb{N}}{a}\end{array}$ & Anmerkungen \\
\hline $\begin{array}{ll}27 / 9 & 2 I \\
28 / 9 & 2 I \\
29 / 9 & 2 I \\
30 / 9 & 2 I \\
1 / 10 & 2 I \\
3 / 10 & 2 I \\
4 / 10 & 2 I \\
5 / 10 & 2 I \\
6 / 10 & 2 I \\
8 / 10 & 2 I\end{array}$ & $\begin{array}{l}\text { Ruhe } \\
\text { Gehen im Zimmer } \\
\text { I mal. Treppensteig. } \\
\text { Ruhe } \\
\text { Ruhe } \\
\text { Ruhe } \\
\text { Ruhe } \\
\text { Ruhe } \\
\text { Ruhe } \\
\text { Ruhe } \\
\text { Ruhe } \\
\text { Ruhe } \\
\text { Gehen im Zimmer } \\
\text { I mal. Treppensteig. } \\
5 \text { mal. Treppensteig. }\end{array}$ & $\begin{array}{r}80 \\
\text { I I } 4 \\
\text { I } 50 \\
\text { IOO } \\
74 \\
76 \\
90 \\
70 \\
94 \\
64 \\
72 \\
80 \\
\text { I } 26 \\
\text { I62 } \\
\text { I } 76\end{array}$ & $\begin{array}{r}62 \\
\text { I } 00 \\
\text { I } 16 \\
84 \\
62 \\
68 \\
86 \\
70 \\
94 \\
64 \\
72 \\
80 \\
\text { I IO } \\
\text { I } 34 \\
\text { I } 24\end{array}$ & $\begin{array}{r}\text { I } 8 \\
\text { I4 } \\
34 \\
\text { I6 } \\
\text { I2 } \\
8 \\
4 \\
0 \\
0 \\
0 \\
0 \\
0 \\
16 \\
28 \\
52\end{array}$ & $\begin{array}{l}\text { keine } \\
\text { Herztonica. }\end{array}$ \\
\hline
\end{tabular}

Tab. VI und Abb. 3 betreffen einen Patienten (J.P. H., Nr. 4 $\mathrm{v}$ on Tab. I), der im ganzen 5 mal wegen desselben Leidens in der Klinik aufgenommen wurde. Die Kurven veranschaulichen das Verhalten des Pulsdefizites während einer Behandlung mit Tinct. strophanti. Nach 6 Tagen ist das Pulsdefizit bei Ruhe, aber nicht nach Anstrengungen, verschwunden. Es erhellt zugleich aus der Tabelle, daß das Pulsdefizit mit zunehmenden Anstrengungen zunimmt (vgl. Tab. III). Das Ergebnis von Untersuchungen an demselben Patienten vom Anfang und Ende eines früheren Krankenhausaufenthaltes findet sich auch in Tab. IV (Nr. 4). Pat. wurde damals mit Digalen behandelt. Es gelang damals gleichfalls, das Pulsdefizit bei Ruhe (praktisch gesprochen) zum Schwinden zu bringen, während nach 5 maligem Treppensteigen ein bedeutendes Defizit vorlag.

Abb. 4 rührt von einer Patientin (J. H., Nr. Io von Tab. I) her, die mehrere Monate (5. VIII. 19 bis 3. I. 20) in der Klinik verweilte, ohne daß es trotz energischer Behandlung sowohl mit Digalen als mit Tinct. strophanti gelang, eine tatsächliche Besserung ihres Zustandes zu erzielen. Es kamen $\mathrm{ab}$ und $\mathrm{zu}$ kurze Perioden vor, in denen der Puls langsam und das Pulsdefizit entweder (an einigen Tagen) ganz verschwunden oder seht klein war. Im großen ganzen lagen die Verhältnisse, wie in Abb. 4 dargestellt; diese Kurven stammen aus dem ersten Teil ihres Krankenhausaufenthaltes. Sie war ab und $z u$ subfebril und hatte während einer kürzerenPeriode leichte Gelenkschmerzen. Sie starb einige Monate nach der Entlassung zu Hause.

Abb. 5 rührt von einem $\mathrm{Pa}$ -

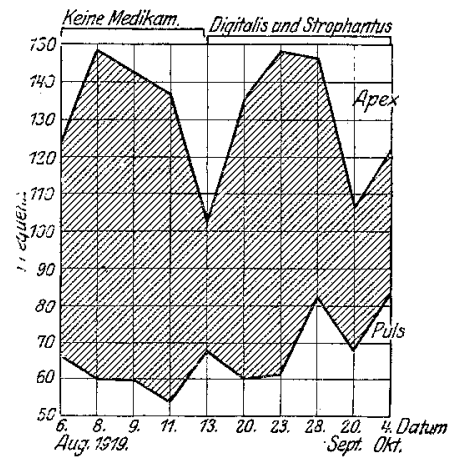

Abb. 4. Graphische Darstellung des Verhaltens des Pulsdefizits bei einem Patienten Tab. I, Nr. ro), bei dem weder Digitalis noch Strophantus das Pulsdefizit zum Schwinden bringen konnte. Pat. starb einige Monate nach der Entlassung aus dem Krankenhause. tienten (C. H., Nr. I6 vom Tab. I)

her, der während eines Anfalles von Lungenödem im Krankenhaus untergebracht wurde. Er findet sich auch in Tab. IV (Nr. 5 ) und in dem entsprechenden Text. Nach einer Vorbehandlung mit großen

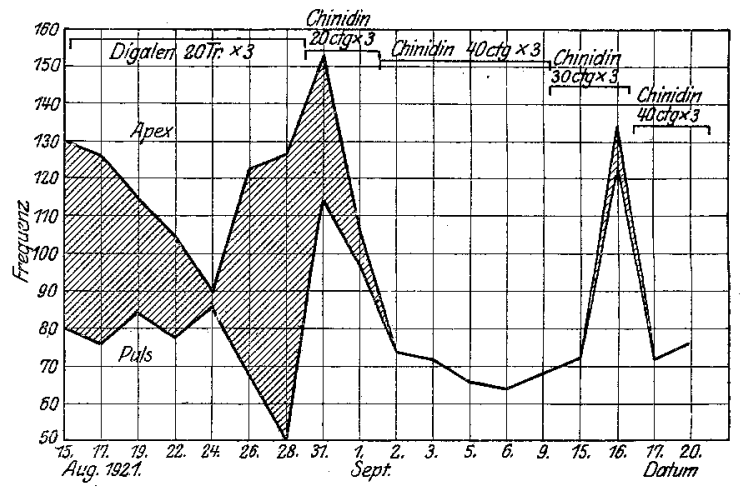

Abb. 5. Graphische Darstellung des Verhaltens des Pulsdefizits bei einem mit Digitalis und Chinidin behandelten Patienten (Tab. I, Nr. I6).

Digitalisgaben wurde Patient mit gutem Erfolg mit Chinidin behandelt. Die Herzaktion wurde während dieser Behandlung langsam, und das Pulsdefizit verschwand. Gleichzeitig wurde, was nicht aus den Kurven zu ersehen ist, die Aktion regelmäßig, indem das vorhandene Atriumflimmern und somit auch die Ursache der Arhythmie aufgehoben wurde. Vgl. die Abb. 2 und 3, wo Digitalis bzw. Strophantusbehandlung anscheinend dieselbe Wirkung auf Herzfrequenz und Pulsdefizit hatten, wo aber die Ursache der Arhythmie fortwährend bestehen blieb und der Puls sich daher unregelmäßig erhielt.

\section{Die Bedeutung des Pulsdefizits für die Prognose.}

Es erhellt aus dem Vorhergehenden, daß das Verhalten des Pulsdefizites von Bedeutung ist für die Prognose quo ad functionem. Von ihrer Bedeutung für die Beurteilung der Prognose quo ad vitam ist noch nichts zuverlässiges bekannt. Wahrscheinlich ist es jedoch ein in dieser Beziehung schlechtes Zeichen, wenn ein Pulsdefizit (bei Ruhe) nicht während einer korrekt geleiteten Behandlung schwindet. (Vgl. Abb. 4.). 